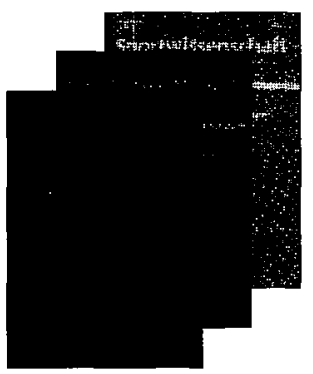

\title{
Vorschau
}

Folgende Beiträge wurden nach Prüfung

durch die Gutachterinnen und Gutachter zur Publikation

angenommen und stehen zur Veröffentlichung

in den nächsten Heften an

Andreas Bund

Laila Ottesen

Alexander Priebe
Selbstgesteuertes Bewegungslernen und Lernstrategien

Sports Participation, Gender and the Welfare State

Olympische Kämpfe - Carl Diem und Otto Peltzer.

Biografische Einblicke in die deutsche Sportgeschichte des 20. Jahrhunderts

\section{Förderpreis für Sportdidaktik 2004}

Den Förderpreis für Sportdidaktik 2004, der zum siebten Mal vergeben wurde, erhielt Dr. Matthias JAKOB mit der Arbeit „Erkennen durch Handeln“. Der „Beitrag zu einer erfahrungsorientierten, praktischen Unterrichtslehre des Sport- und Bewegungsunterrichts" soll die Lücke, die zwischen Theorie und Praxis des Sportunterrichts klafft, schließen. Die Arbeit belegt, dass aus der reflektierten Praxis erfahrungsbezogene wissenschaftliche Erkenntnisse als Grundlage einer praktischen Unterrichtslehre gewonnen werden können.

Der Förderpreis (500 Euro) wird auch 2006 wieder vergeben. Die Jury bittet, Arbeiten, die praxisbezogen Erziehungswirkungen und -möglichkeiten des Sport- und Bewegungsunterrichts untersuchen, bis zum 30. November 2005 an die Erziehungswissenschaftliche Fakultät der Universität Erlangen-Nürnberg, Lehrstuhl für Sportpädagogik, Regensburger Straße 160, 90478 Nürnberg, zu senden. Ausschreibung und weitere Auskünfte sind dort ebenfalls erhältlich. 